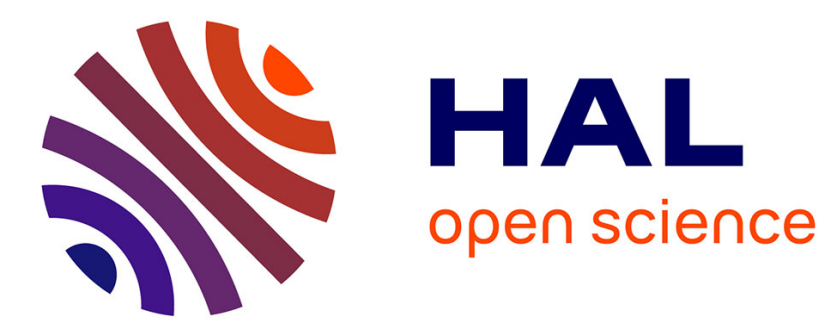

\title{
Nouvelles fouilles à Lezoux (Puy-de-Dôme)
}

Aline Mathonnière-Plicque, Albert Grenier

\section{To cite this version:}

Aline Mathonnière-Plicque, Albert Grenier. Nouvelles fouilles à Lezoux (Puy-de-Dôme). Gallia - Fouilles et monuments archéologiques en France métropolitaine, 1961, 19 (1), pp.55-69. 10.3406/galia.1961.2315 . hal-01926107

\section{HAL Id: hal-01926107 \\ https://hal.science/hal-01926107}

Submitted on 25 Feb 2020

HAL is a multi-disciplinary open access archive for the deposit and dissemination of scientific research documents, whether they are published or not. The documents may come from teaching and research institutions in France or abroad, or from public or private research centers.
L'archive ouverte pluridisciplinaire HAL, est destinée au dépôt et à la diffusion de documents scientifiques de niveau recherche, publiés ou non, émanant des établissements d'enseignement et de recherche français ou étrangers, des laboratoires publics ou privés.

\section{(이) $\$$}

Distributed under a Creative Commons Attribution - NonCommercial - NoDerivatives 44.0 


\title{
NOUVELLES FOUILLES A LEZOUX (Puy-de-Iôme)
}

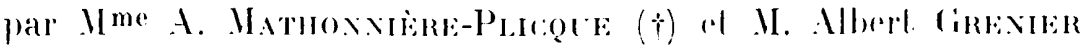

Au moment, où j'allais domer i l'impression cet article qu'avail bien voulu m'envoyer la fille du Dr Plicque, le grand fouilleur do Lezoux, Mme Mathonnière, j'ai eu le regret d'apprendre sa mort inopinée. Je n'en ai pas moins eru devoir donner dans Gallia ces notes de première main, non sans les aroir tant soit peu retouchées, ce qui explique mon nom à côté du sien à la fin de l'article. Les déchiffrements ont été vérifiés par mes amis : MII. Robert Marichal, spécialiste de la paléographie et Pierre Fournier, Directeur de la circonscription qui est allé voir sur place les originaux. Je leur suis reconnaissant de l'assurance qu'ils m'ont ainsi donnée. Ise Dr Plicque avait publié peu de choses sur ses fouilles. Ces notes de Mme Mathonnière prennent ainsi la valeur d'un document original'

Je cède ici la parole à Mme Mathonnière.

Dans le jardin même de notre maison de Lezoux (plan cadastral 965 et 966), mon père avait découvert un four de potier. In heureux hasard m'en a fait découvrir un second en 1933, à une profondeur de $1 \mathrm{~m}$. 5ó. Ine nombreuses briques et tuiles de la voûte du four gisaient, uffondrées dans l'intérieur. Les pierres et les briques, vitrifiées par la chaleur gardaient de belles teintes de noir, de vert et d'ocre. L'alandier s'ourrait vers le Nord ; des débris de charbon de bois y gisaient encore. La partie sud, bien enfoncée dans la terre, avail gardé sa solidité. Lin mur de briques délimilait une chambre en avant et sur une parlie des côtés du four ; elle mesurait bे et 6 mètres de côté ; trois marches d'escalier y descendaient du terrain environnant (fig. 1).

Diamètre exlérieur du four : 1,65; ; intérieur : 1,35; largeur de l'alandier : 0,45. La sjle qui devait séparer l'alandier de la chambre de cuisson du four avait complètement disparu mais les consoles qui la portaient en montrent le niveau à 0,47 au-dessus du foyer. De la chambre de chauffe ne subsistait que le bas des murs mais le long de ces murs s'élevaient des tnyaux partant du foyer; j'en ai recueilli des restes rolorés en rouge ou violacé par l'efiet de la chaleur. C'étaient des tubes qui devaient s'emboîler les uns dans les autres.

(1) L'essentiel des trouvailles de Dr Plicque a été publie par J. Déchelette dans le chapitre Lezour.r de son ouvrage Les Vases céramiques mrnes de la fiaule ronaine 1901 , I, p. 138-203, dapres la partie des collections du Docteur qui ont ahouti au Ifuse des Antiquites nationales de saint-Germain-en-Lave. Sur les collections du Dr Plique et un manuscrit de lui demeuri inédit que conserve le .lusée, voir Dr Doranlo, La collection Plicque an .Musée de SaintGermain, dans Velanges $G$. Radel, Rer. Eludes anciennes, 1910, p. 613-621. I'ne autre partie des collections avait. abouti au British Museum, d'ou II. B. Walters, Cataloque of the Roman Pollery in the Depariment of Anliquilies of the Brilish .Museum et, en dernier lieu : J. A. Stanfield et Grace Simpson, Cenlral Gaulish Pollers, Oxford, 1958. Voir aussi S. Reinach, Calalogue illustré du . Yusee des Antiquiles nalionales. I (1917. p. 213-215) II 1921) p. 136-114. 


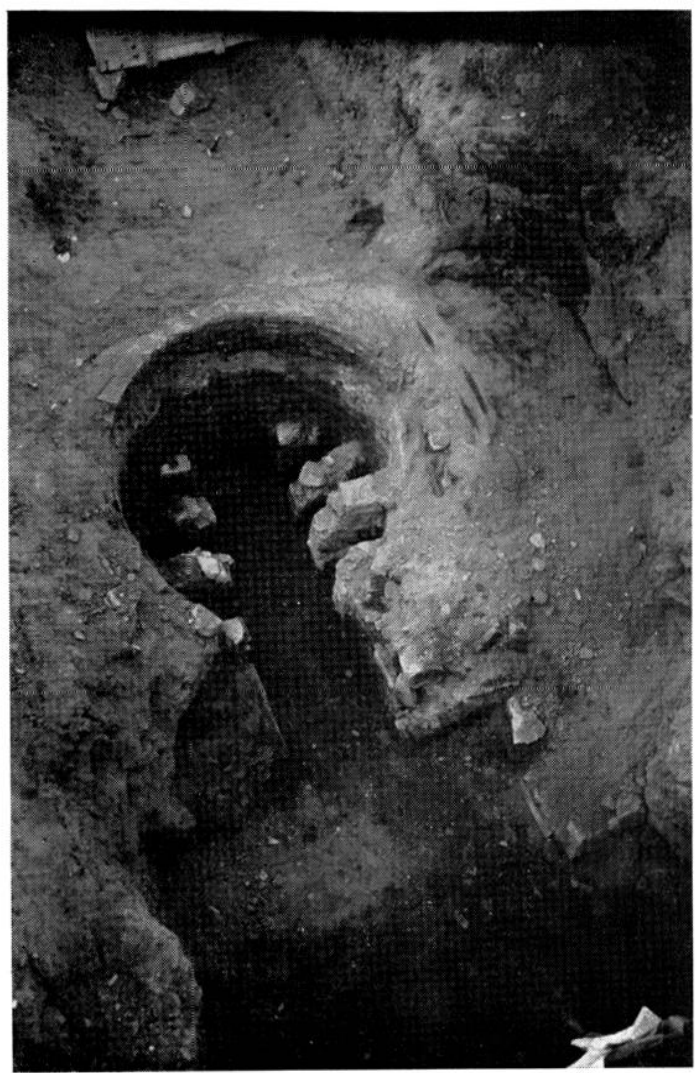

Fìr. 1. Le four an jardin de I.e\%oux.

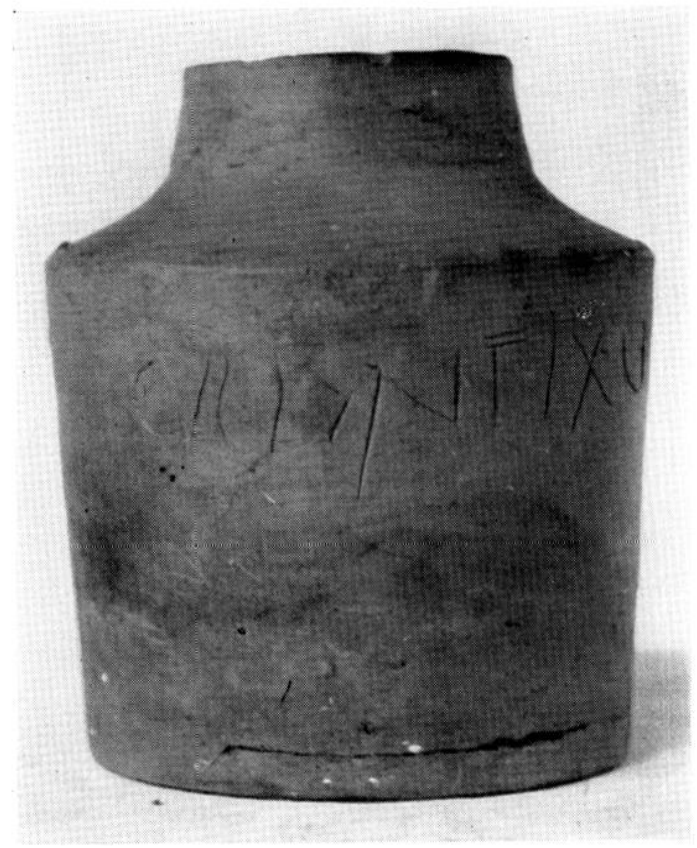

Fig. 3. ... Le tuyau de chaleur avec graffite. Les dimensions sont les mêmes que celles du tụauu fịg. ?. Voir fig. 1.

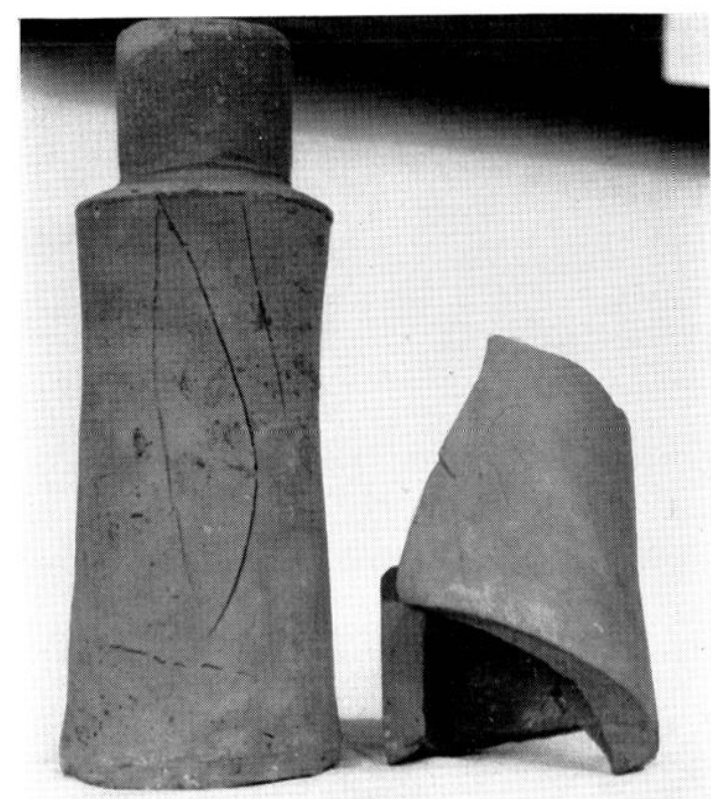

Fig. 2. In des tuyaux de chaleur du four.

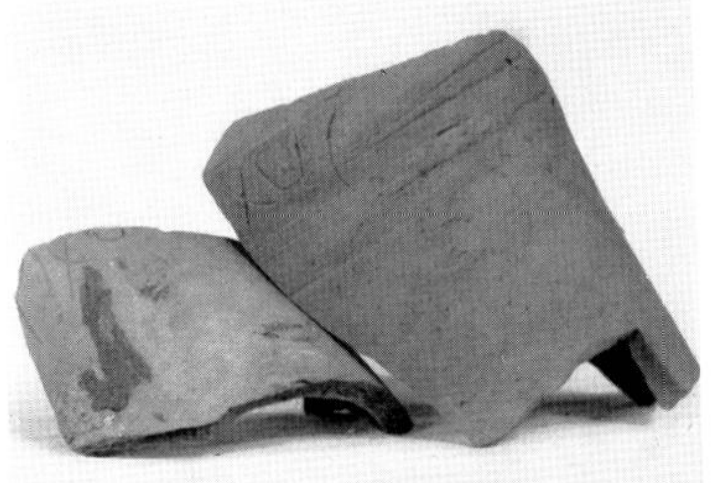

Fig. t. Fragment de turan avec la fin du graffite: i.rus. Voir fig. 3. 
L'un, qui était entier, mesurait 0,21 de hauteur, 0,08 de diamètre et autant pour l'épaisseur de la terre. Il était orné d'un dessin et peut-ètre de quelques lettres, absolument indéchiffrables (fig. 2). Ln autre, de mêmes dimensions, porte en grands caractères cursifs un graffite qui se lit Orantixus (fig. 3); sur un autre fragment se lit la finale ....ixus (fig. 4) et le nom se trouve confirmé de la façon la plus nette par de nombreuses estampilles imprimées sur des tessons trouvés aux alentours. J'ai cru powvoir supposer que cet, Orantixus était le potier propriétaire du four.

Sur l'avant du four un fragment de béton ou d'enduit portait un autre graffite que M. Marichal lit Rufius Veneroni et qui échappe à toute interprétation (fig. 5).

Autour du four j'ai trouvé de nombreux restes des supports destinés à isoler les piles de vases de la chaleur excessive qui se dégageait des ouvertures de la sole. Ces supports consistent en un trone de cône évidé avec un rebord, plutòt qu'un bourrelet, à paroi légèrement incurvée; la hauteur varie entre 0,04 et 0,05 ; diamètre du haut de 0,65 à 0,95 ; diamètre du pied, entre 0,07 et 0,08 . La terre en est de couleur pâle. Pas d'autres cales que de curieux petits objets d'argile de forme identique et à peu près de mêmes dimensions : 0,30 et 0,$34 ; 0,019$ de haut ; 0,007 d'épaisseur. Faits de la pâte des fragments de céramique, res morceaux d'argile de forme trapézoïdale portent quelquefois la trace des godrons ou dessins des vases contre lesquels ils étaient appliqués. Ces curieuses cales de potiers étaient restées inconnues jusqu'à ce jour. J'en ai recueilli une soixantaine. (Rien ne prouve que des tessons catalogués simplement tessons n'aient, comme ailleurs, servi de soutiens des piles de vases dans le four) (fig. 6 et 7 ).

Parmi les déblais, dans le fond du four, j'ai eu la surprise de ramasser la reproduction en terre cuite d'une noix, parfaitement imitée et de grandeur naturelle. La couleur même donnait l'impression de la réalité. Périmètre : 0,08 ; haut. $0,002 j$ (fig. 8).

Après extraction de tous les débris, le four dut être comblé.

\section{Céramique.}

Parmi les débris du four et aux alentours j'ai recueilli de nombreux tessons. Ils étaient d'aspects divers, le plus grand nombre en pàte tendre, non vitrifiée, de couleur blanchâtre, correspondant à une cuisson peu poussée, avec quelques parcelles de mica, d'autres de couleur noire ou grise et quelques tessons de sigillée rouge. Dans les notes de mon père sur ses essais de classification, je trouve indiqué que les pâtes tendres ont précédé les pâtes dures; on a cessé à Lezoux de fabriquer des poteries roses, blanches, grises ou noires lorsque les poteries rouges se sont montrées.

\section{Pâtes lendres.}

Les différences de couleur ne résultent, sans doute, que des circonstances accidentelles d'une cuisson imparfaite; il convient de n'en tenir compte que pour les essais qui peuvent ètre tentés pour reconstituer les rases ; c'est pourquoi je les indiquerai.

Céramique blanche. Très nombreux fragments anépigraphes. J'ai pu reconstituer un vase du type de l'olla, magnifique par son élégance et sa légèreté (fig. 9). Corps presque 


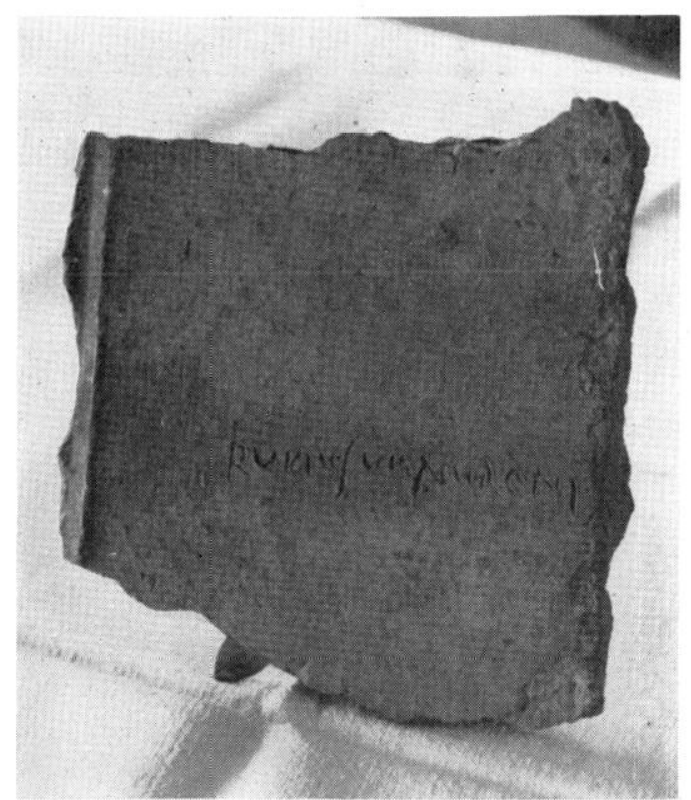

liig. 5) a. .. (iraffite sur le ciment du four.

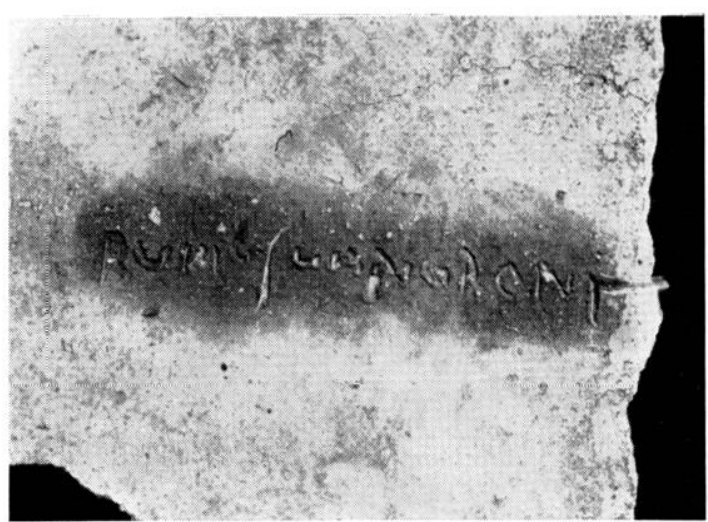

Fig. 5 b. Graflite sur le ciment du four. Détail.

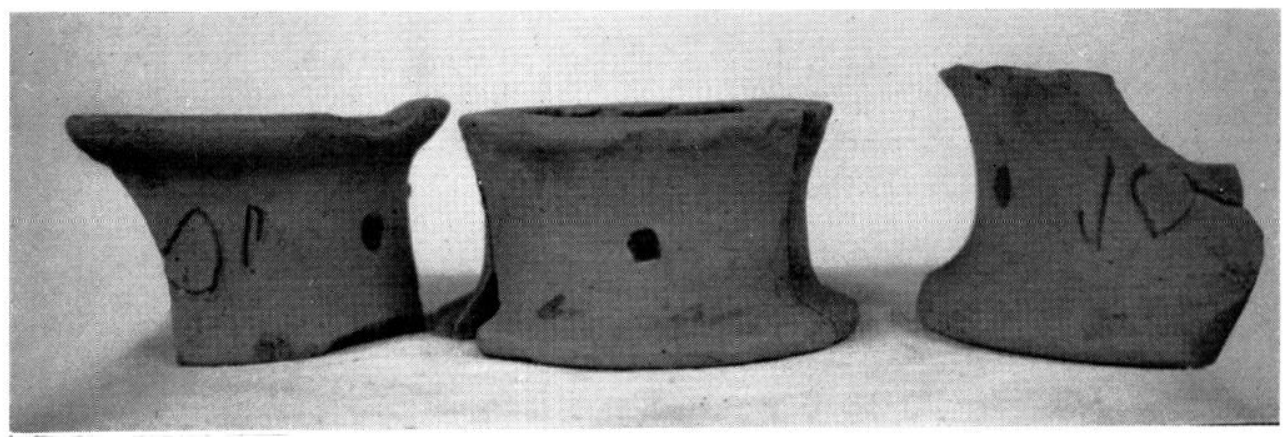

Fig. fi. Supports des piles de vases dans le four.



Fig. 7 Cales des piles de vases dans le four.



Fig. 8. - Noix en terre cuite trouve dans le four. 
sphérique ou ovoüde avec deux anses latérales unissant l'épaule à un col étroit et allongé bordé d'un léger rebord. Le vase repose sur un petit pied. Hauteur 0,38; diam. 0,70; épaisseur des parois 0,002 . Le type se rapprocherail, mais avec un col beaucoup plus étroit, de celui de Dragaendorff, pl. III, 53, qui a les deux anses ou, plutôt, de la figure de Déchelette, Vases ornés, I, pl. II, no 60, qui n'a qu'une anse. Il remonte à la céramique de La Tène I où il est fréquent mais sans anses².

Assiettes plates (types Dragendorff, pl. I, $n^{\text {os } 1-3 ; ~ I I, ~ n o s ~ 17-21) . ~ D e ~ t e i n t e s ~ d i f f e ́ r e n t e s ~ ; ~}$ rebords très variés et tailles diverses, diam. de 0,14 à 0,26 . Estampille au centre intérieur.

Coupes très petites, tessons fort nombreux, guillochées sur le bord intérieur; diam. 0,06 a 0,08 . Estampille au centre intérieur (fig. 10). Bols, forme Drangendorff 24-27; guillochés sur la bordure extéricure. Estampille au centre intérieur. Diam. 0,10 à 0,12 (fig. $10)$.

Terre noire : une coupe à grandes anses latérales (I)rag. pl. III, 4:) . Fragments de vase orné (Drag. 29), vase caréné, type ancien. Tessons de vases carénés, ornés de guillochis et de zones d'impressions a la molette. Pieds développés (fig. 11 et 12).

Terre grise : mèmes bols qu'en terre blanche (Drag. 24-27) el même coupe peu profonde à grandes anses latérales qu'en terre noire (I)rag. 42). Tessons de coupes type 29 carénées ou godronnées, le plus souvent avec: des fragments de frises décoratives excellentes : fig. 13, 14, 15. Fig. 16, deux grands fragments d'un vase godronné. Fig. 17 : frise



Fig. 9. - Olla en terre blanche, reconstituée. supérieure d'un vase caréné.

Dans cette décoration les figures demeurent exceptionnelles : rares figures d'animeux héraldiques, plus rares encore, de gladiateurs.

\section{Moules.}

Les trouvailles se sont trouvées complétées par une série de moules de même terre et de même fabrication que les vases. Ils sont d'un travail excellent et présentent tous les caractères du premier siècle : 



Fï. 10. Types Dragrendorff, pl. II et III.

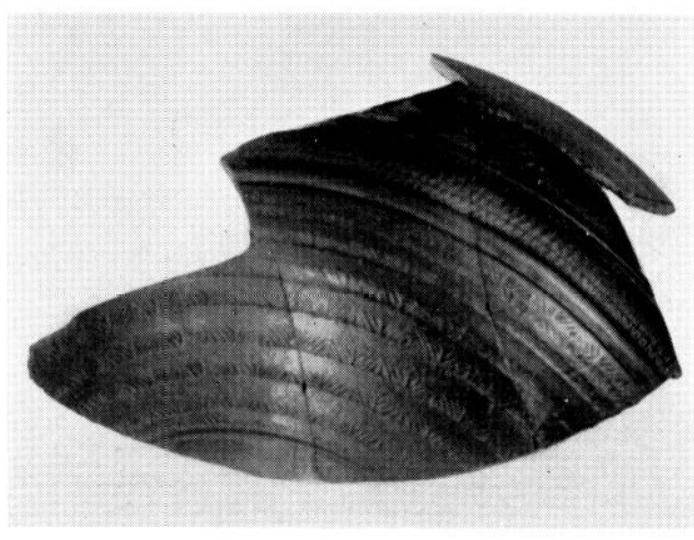

Fir. 11. -. Tesson de vase orné de zones estampies à la molette.

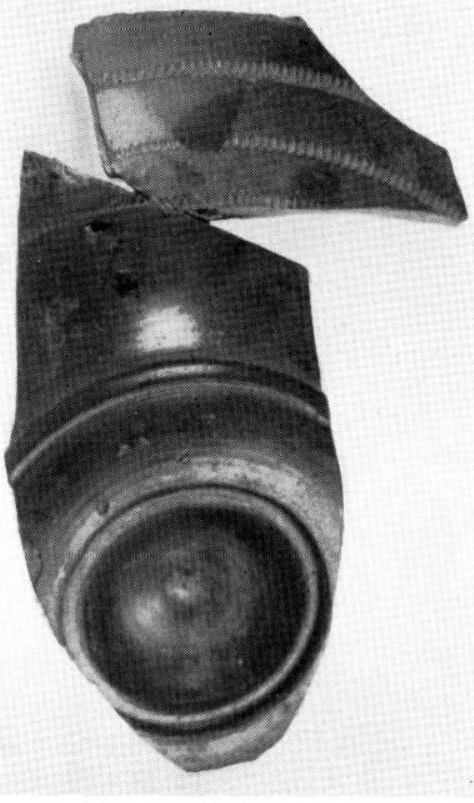

Fig. 12. - Tessons avec zones quillochées. 




Figr. 13. - Fragment carené avec frise décorative.
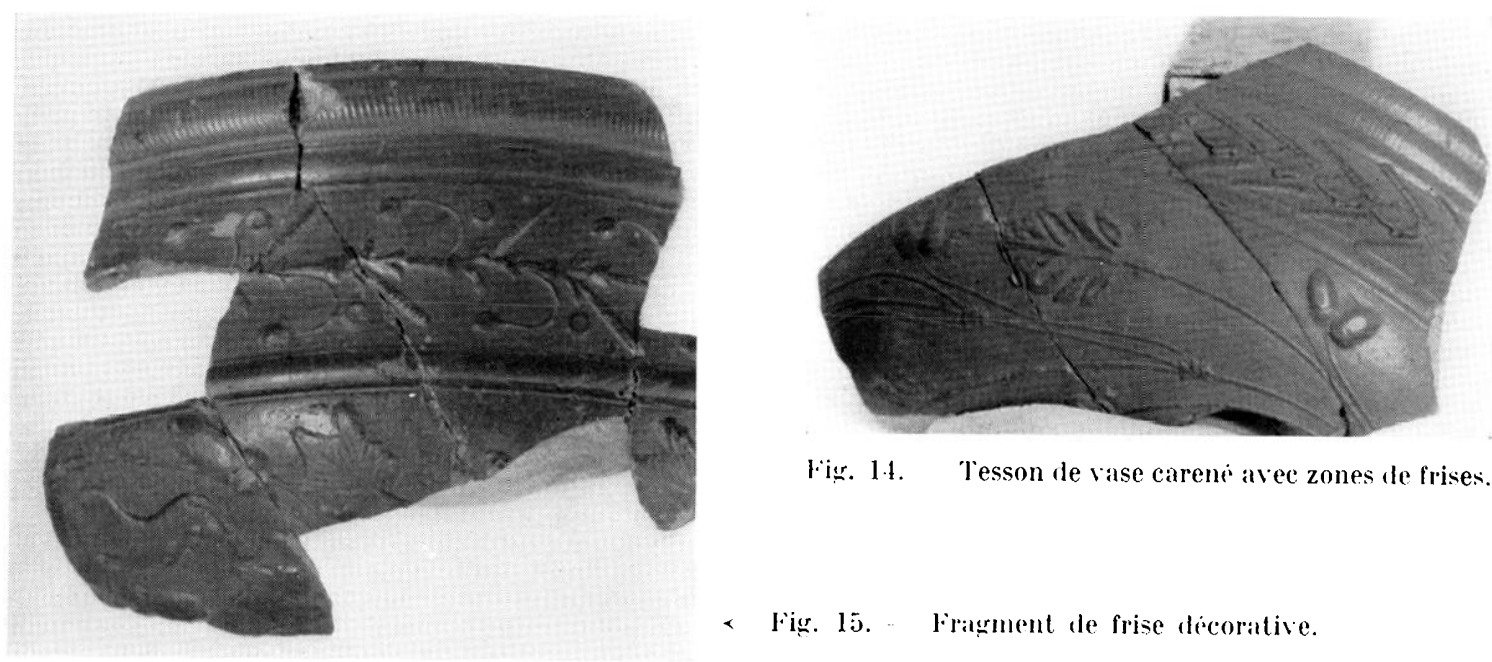

Fig. 14. Tesson de vase carene avec zones de frises.

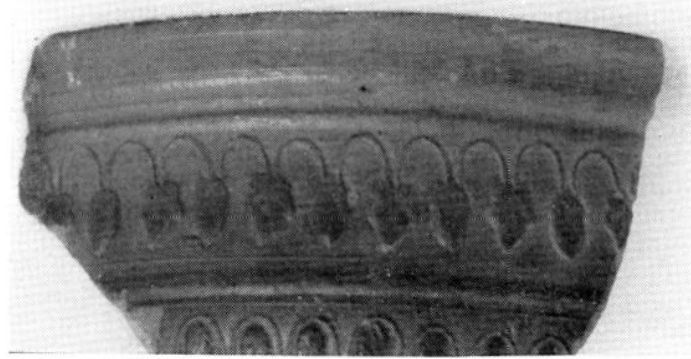

\& Figr. 15. Fragment de frise dicorative.

l:ïr. 17. - Frise d'un vase caréné.

Fir. 16. - Fragments de grande coupe grodromie. -.>

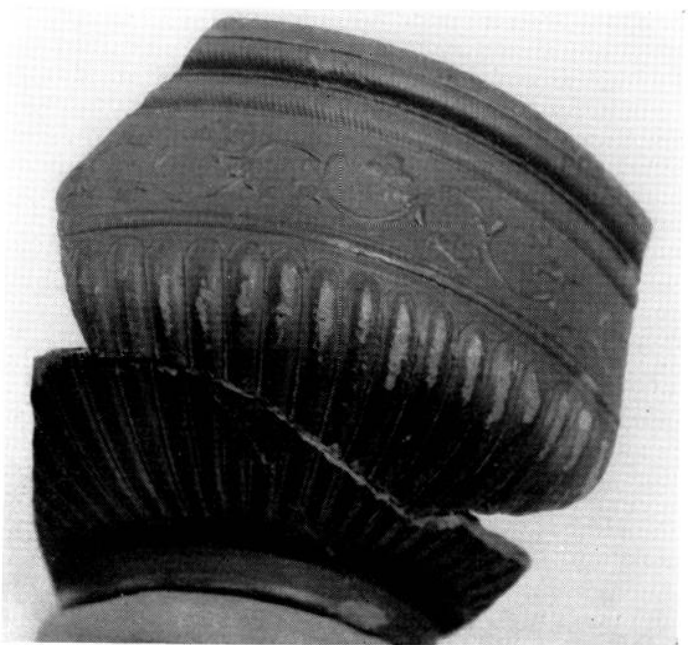






Jï. 1R. Fond de moule.



Fïg. 19. - Franment de moule arec ornements.

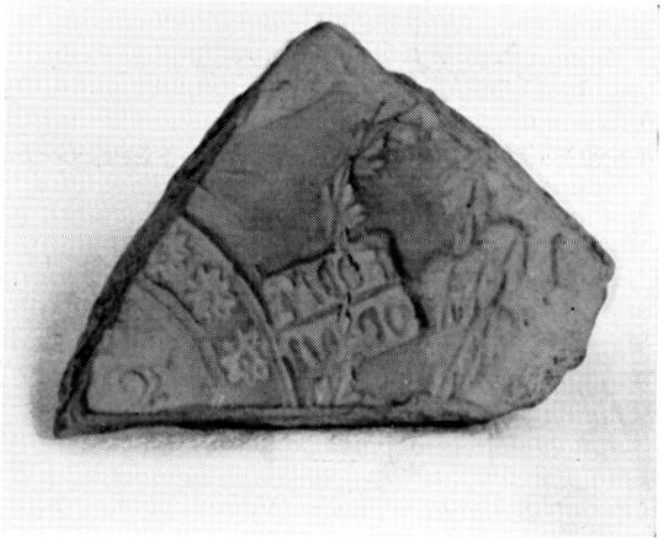

Fig. 21. Sur moule, inscription ornce inversée.



Figr. 20, . Frarment de moule avec ornements. 


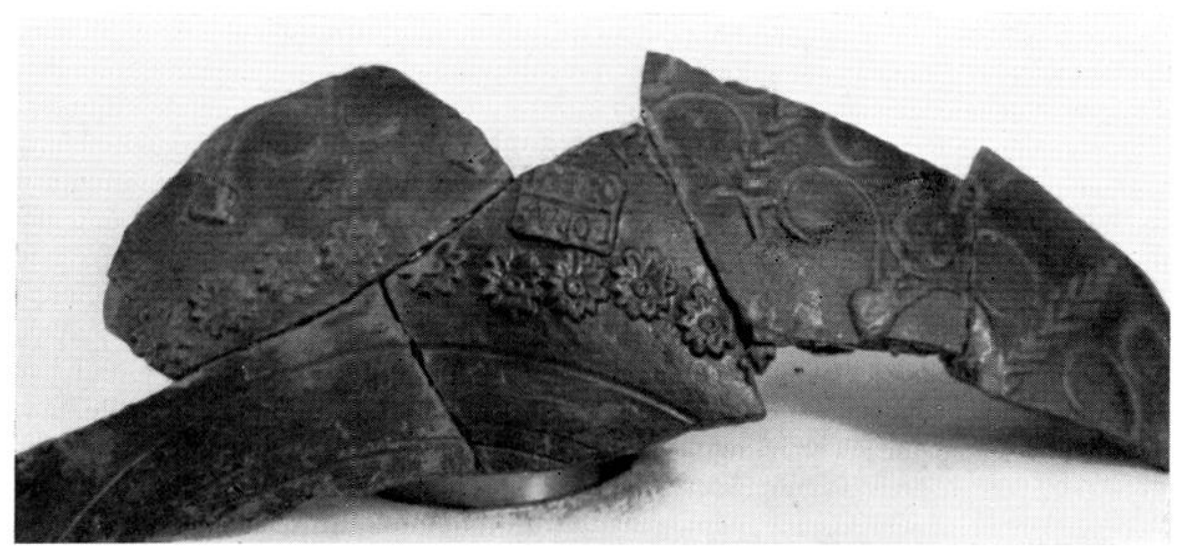

Fig. 22. - Sur moule, inscription FORMA/OPIANI.

Fig. 18. Moule d'un fond de vase.

Fig. 19 et 20. Fragments de moules de frises décoratives.

Parmi ces fragments de moules, plusieurs portent une estampille inversée destinée à venir s'imprimer dans les ornements du vase. M. P.-F. Fournier a bien voulu aller vérifier les lectures.

Fig. 21. Dans un cartouche rectangulaire apposé perpendiculairement à un cercle garni de petiles rosaces : estampille inversée; 1 re ligne: forma ( $M$ et $A$ liés); 2 e ligne: Opiani ( $A$ et $N$ liés; la fin des mots contre le cercle des rosaces).

Fig. 22. Autre fragment de moule donnant la même estampille inversée mais dans un cartouche parallèle à un cercle orné de rosaces.

Fig. 23. Fragment de vase donnant. l'épreure positive de ce moule (se voit dans le 'arl ouche reclangulaire superposé au cercle des rosaces) : forma Opiani.

Fig. 24. Au centre d'un fond de vase, inseription sur deux lignes en lettres saillantes qui ne sont pas inversées. Ligne supérieure, en lellres plus petites : Dura (le $D$ inilial peu visible sur la photographie m'est allesté par M. P.-F. Fournier); deuxième

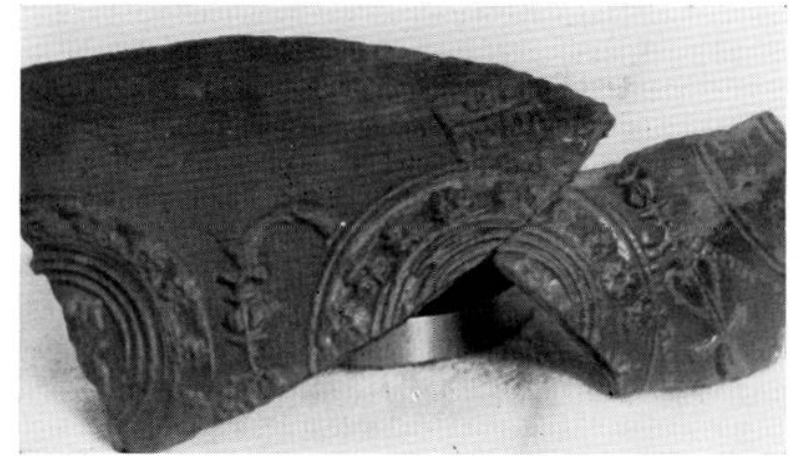

Fig. 23. - Dans un cartouche, au-dessus du cercle, la même estampille. ligne: $F$ suivi d'un point en haut et Are; si les lettres n'étaient saillantes, l'estampille pourrait être prise pour une marque de potier plutòt que pour un moule; elle s'inscrira en creux et inversée dans le fond du vase. Les lettres de la seconde ligne sont près du double de celles de la première.

Fig. 25 et 26. Deux noms identiques sur une ligne inversée: Titos en grosses lettres fortement saillantes, sous la guirlande de la frise médiane el, aulre figure, au-dessus de la frise du fond. Les estampilles sur deux lignes séparées par un trait paraissent inspirées des modèles d'Arezzo où elles sont courantes. Il serait cependant trop hardi de penser à Are(tium) pour le dernier groupe de l'inscription fig. 24.

Toute cette céramique, tessons de vases ou moules, relève de la période ancienne des fabrications de Lezoux, antéricure à celle de la sigillée. La date, d'ailleurs, se trouve confirmée par la présence d'une moitié de vase de céramique peinte datant, au plus tard, du début du premier siècle de notre ère (fig. 27 ). 




Figr. 24. - Estampille dans un fond de vase DI RA/L. ARl:.

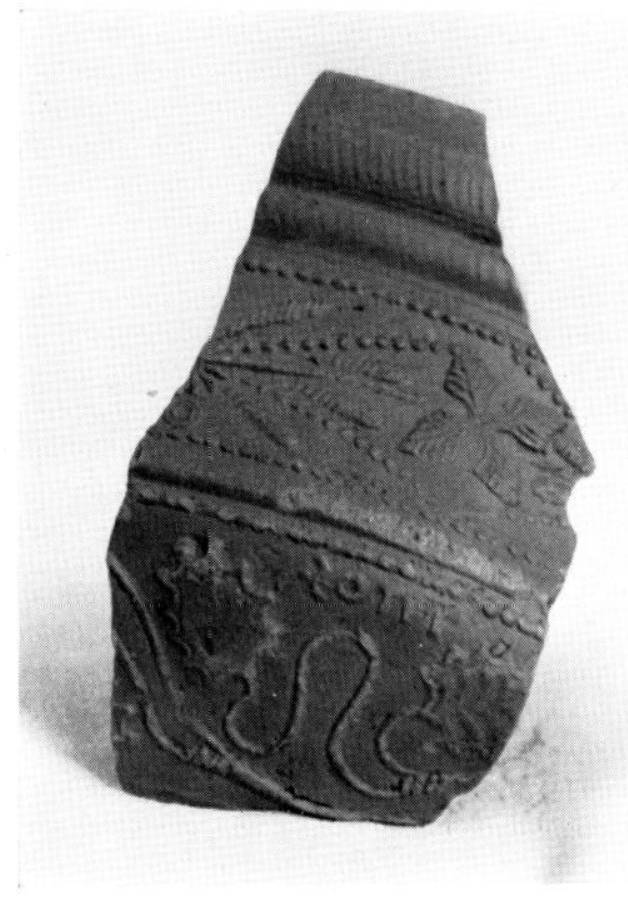

liig. 26. I.'estampille TITos inversie dans la decoration du bas de vase.

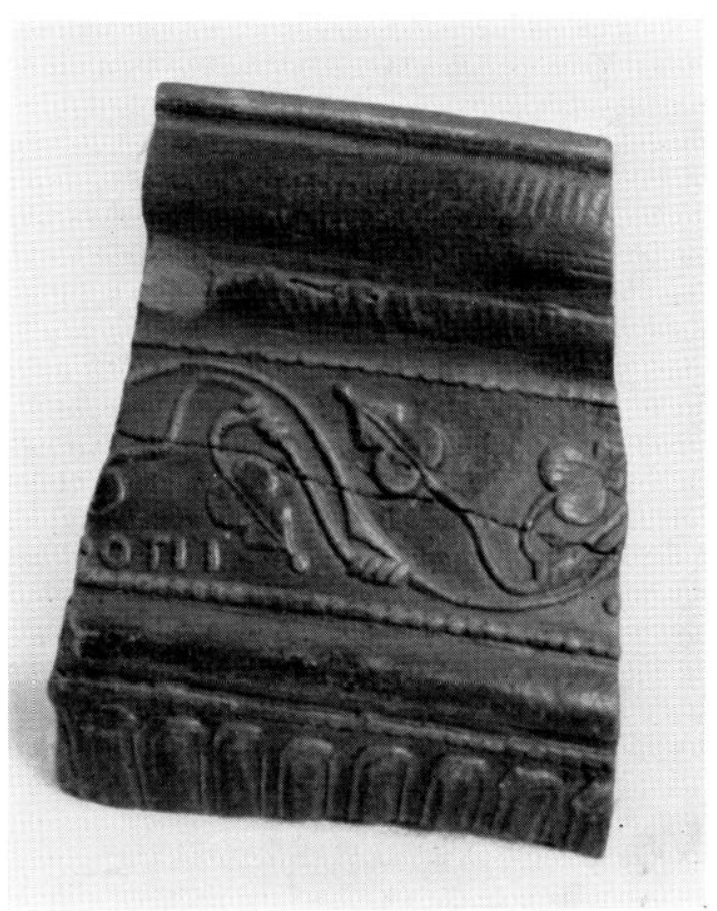

Fig. 25. L'estampille TITOS inverse.e.

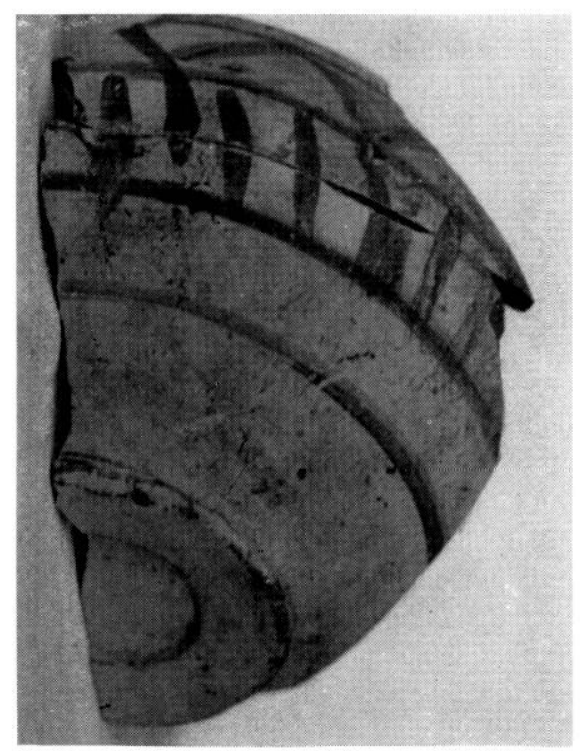

Fig. 27. Fragment de céramique peinte. 
Sur un fond blanc limité par un trait cireulaire vient, vers le haut du vase, une zone de gros traits épais tracés au pinceau. On connaît à Lezoux la série des vases peints, rouge ou noir sur engobe blane. Elle se rattache à la tradition de La Tène el semble avoir marqué la production de Lezoux au début de l'époque romaine ${ }^{3}$. Il faut, dit-il, laisser à la période gauloise et au commencement, de la période romaine des vases peints a engobe blanc et

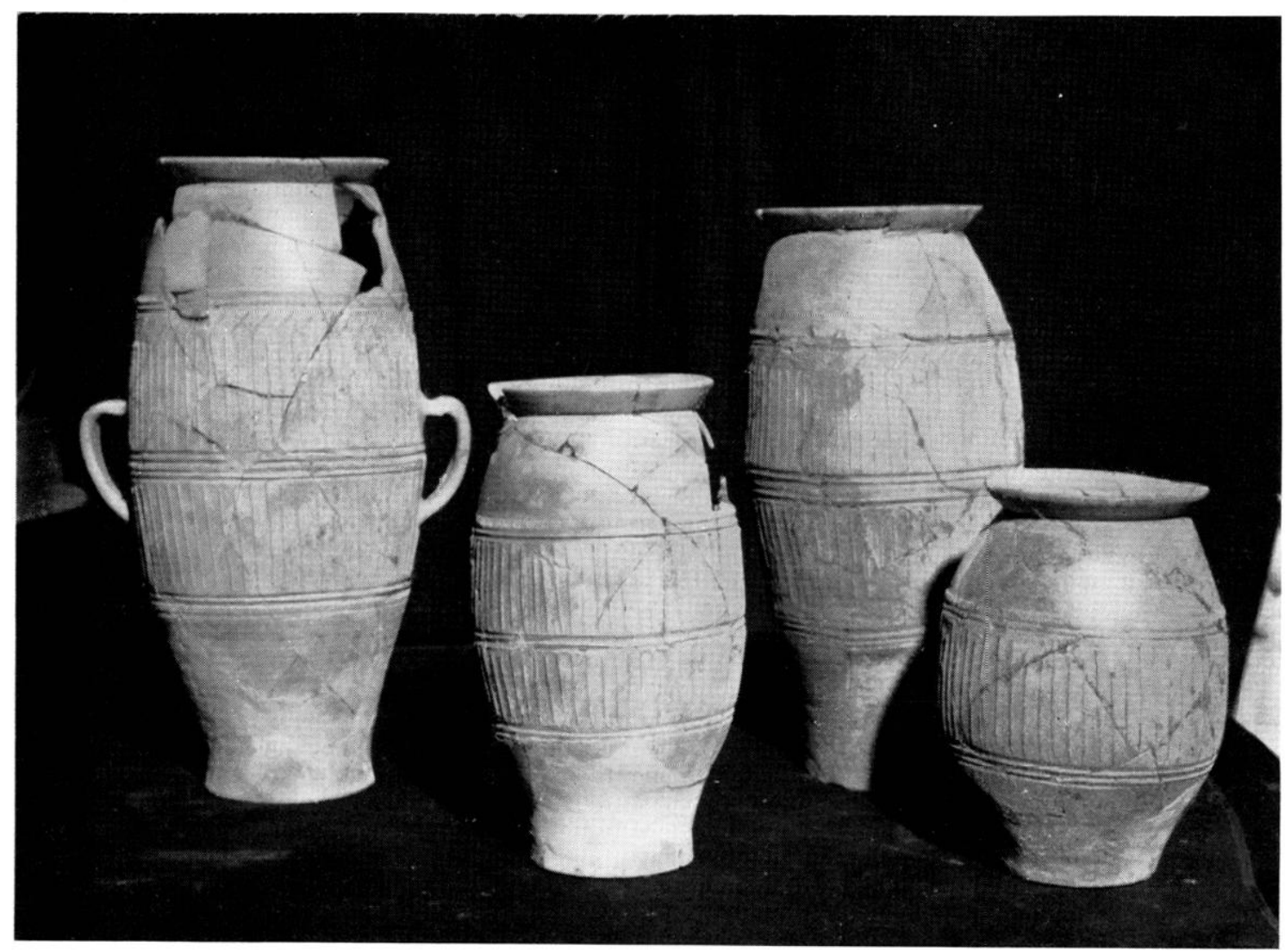

Fig. 28. - Jarres ovoïdes ornees de stries longitudinales.

a décor géométrique qui, par leur technique, leurs formes, leur pâte et leur décoration, diffèrent complètement des poteries romaines. Il y en a, au Musée de Roanne, quelques exemplaires trouvés dans les environs. Déchelette se réservait de les étudier dans le futur volume de son Manuel dont sa mort héroïque en 1914 a privé la science. En résumé, disait-il, la fabrique de Lezoux ainsi que celle de Montans ont livé au commerce des poteries peintes de technique gauloise avant de produire des poteries rouges imitées de celles d'Arezzo. Il n'est d'ailleurs pas impossible que durant cette courte période, la première moitié du premier siècle, les deux techniques aient été exploitées simultanément. 


\section{Grands récipients.}

A une trentaine de mètres de celte officine, sans que je puisse dire si elle en dépendail, j'ai trouvé, en janvier 1957, à deux mètres de profondeur, une masse de celte même céramique grise ou noirâtre mélangéc de quelques lessons blancs. J'y ai complé plus de 2000 tessons dont quelques-uns assez grands. J'eus l'idée de lenter quelques restitutions

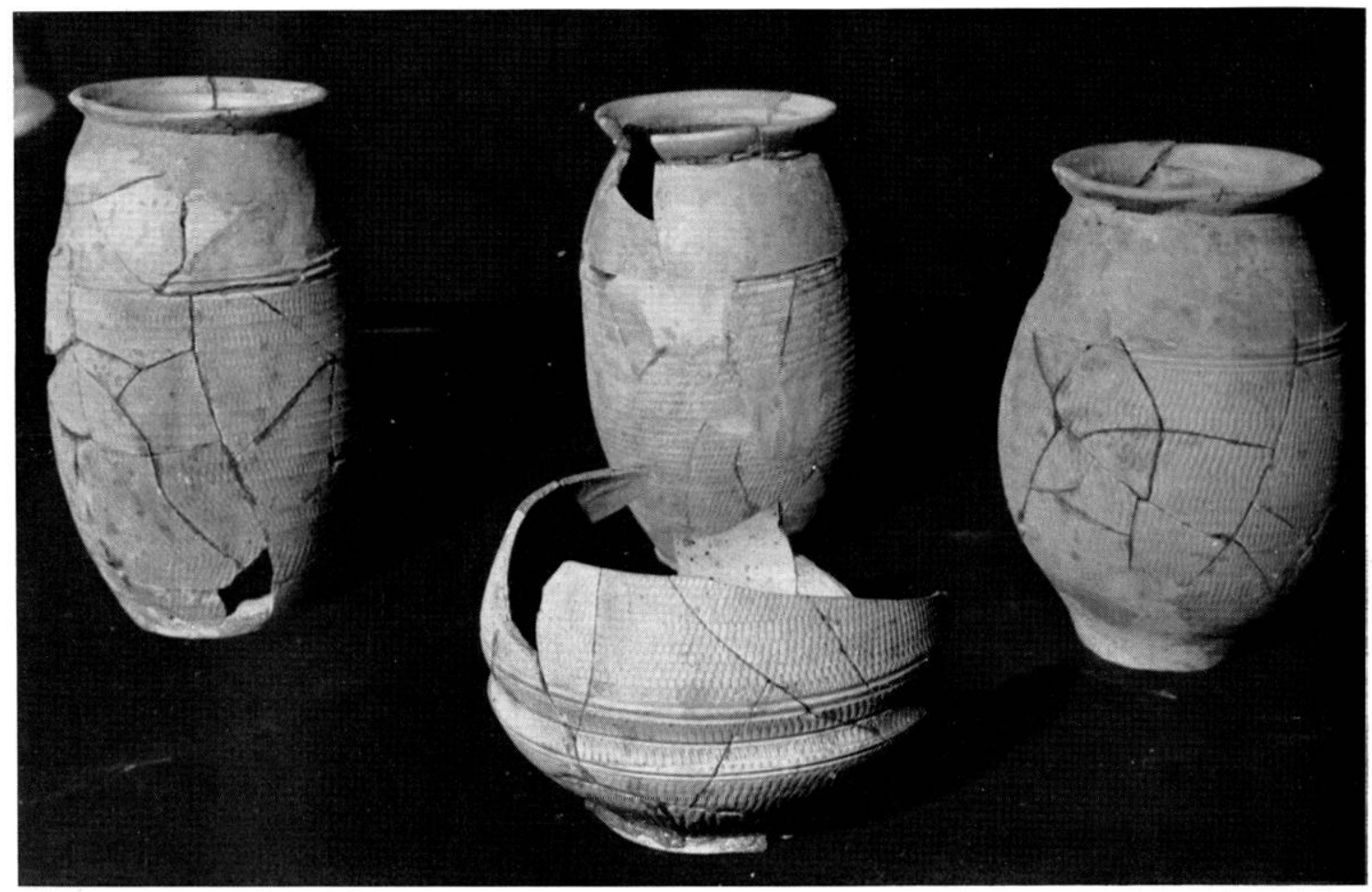

Fig. 29. - Jarres ornées de zones de traits imprimées à la roulette.

et la récompense de ma patience de restauratrice a été la série d'une douzaine de beaux vases du même lype (fig. 28-30)4. La forme est celle d'une olla au corps sphéroïde allongé dont la hauteur varie de 0.25 à 0,34 et le périmètre à peu près des mêmes dimensions; l'épaisseur des parois est de 2 à 3 millimètres; elles sont sonores, a cassures nettes de la couleur de la terre bien cuite. Cinq de ces vases sont ornés d'une ou plusieurs zones de côtes verticales en relief faites d'une barbotine qui a été régularisée à la spatule sur l'engobe. Le lype dérive nettement de la polerie de La Tène ${ }^{5}$ (fig. 28).

Deux de ces vases sont pourvus de deux anses fixées vers le milieu du corps (fig. 28 et 30). Des cercles de rainures horizontales bordent ou interrompent les traits verticaux. Dans le bas de quelques vases apparaissaient plusieurs zones de décor imprimé a la roulette, les mêmes, reconnaissables sur cinq vases à un défaut au dixième trait oblique. Sept autres

(4) Je crois pouvoir les reproduire ici hien que $1 I^{\mathrm{me}}$. Mathonniire les ait déjà publiés mais sans commentaire

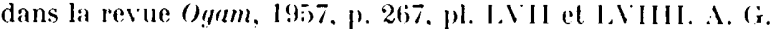

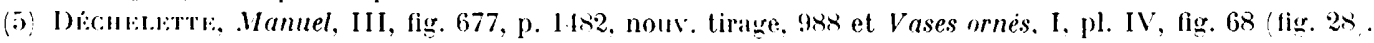




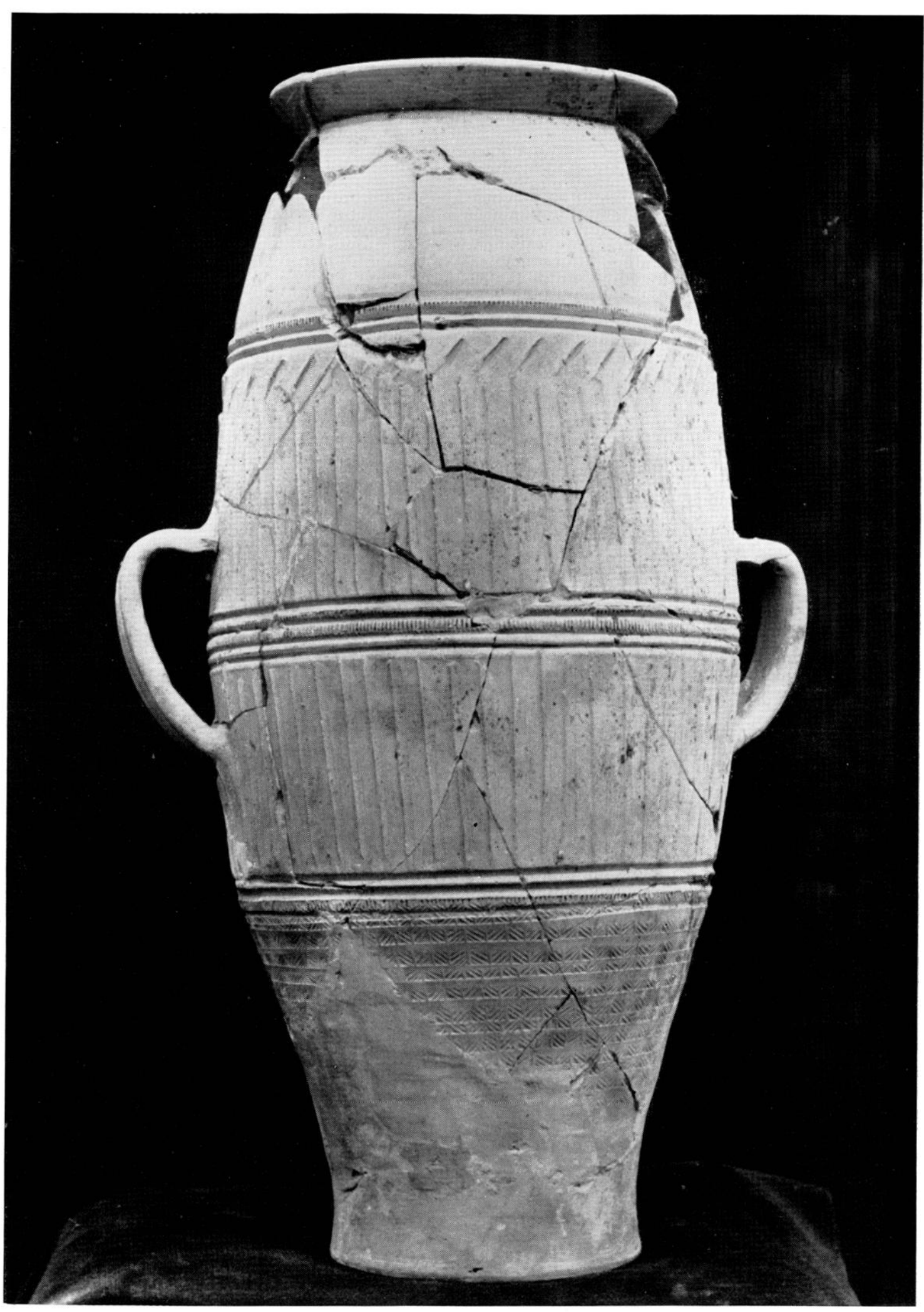

Fig. 30. -- Grand vase unissant les deux types de decorations. 
vases sont entièrement ornés à la roulette (fig. 29). Pour tous ces vases, le bord du col, débordant de plus d'un centimètre, allège la forme; sur ceux qui sont décorés à la roulette, ce bord est agrémenté d'un repli qui n'existe pas sur les autres. Deux de ces rases, d'une hauleur double, et arec deux anses, unissent un décor de rainures verticales à une zone de traits obliques en haut et de nombreuses zones de guillochis imprimées à la roulette dans le bas (fig. 30). Ce procédé de décor imprimé à la roulette est courant en Gaule dès La Tène III'. Quelques fragments de ces mêmes vases se trouvaient d'ailleurs mêlés aux débris de l'officine du four de mon jardin. Il y a là une fabrication qui se rattache à la tradition indigène. Les jarres de ce type abondaient à Bibracte dans tous les ateliers. Elles pouvaient contenir l'eau destinée à tremper le fer ou à tout autre usage.

\section{Marques de poliers.}

Outre ces très nombreux tessons, les recherches autour de mon four m'ont livré quelques estampilles de potiers. Je ne donne ici que celles que j'ai trouvées moi-même ou qui l'ont été sous mes yeux. Je remercie M. P.-F. Fournier qui a bien voulu vérifier mes lectures. Pour les marques connues je renvoie au précieux Index d'Oswald. Cette liste ne représente qu'une petite partie des marques que je possède, venant de la collection de mon père mais dont rien ne me permet d'indiquer la provenance exacte.

1. AETAXl, AITTX. MITAXUs : un fragment trouvé à Moulins, Lezoux, sans date.

2. AETTS.F. Cilé à La Graufesenque, période flavienne.

3. ANTIOGLs. Nom rite dans Oswatr.

1. ARID.F. [A]RIDCOS. : Ardacus a La Graufesenque, époque Tibere-Glaude. Oswald cile: aussi un homonyme ayant I ravaillé à Lezoux aver Seniserus (ci-dessous), sans dale.

5. AVETl $s$. (T et V liés); AVIITl s. sants lieu ni dale (Oswald).

6. BLANIIN. Irzoux, époque de Néron.

7. C.AMLIO. ( $A$ et $M$ liés); C.AMLLO (A, M. V, liés). Estampille fréquente, interprétée par Oswald Camul(ini) O(fficina). Mais j'ai trouvé une fois CAMLLOGE $(A, M, V$, liés), ce qui ferail penser à un Camuloge(nos).

8. COBNERTCS $(N, E, T, V$, liés). Signature fréquente et bien connue à Lezoux, époque Vespasien-Hadrien. J'en rapprocherais une estampille : OBNER. T ( $O$ pointé au centre, $N, E$, liés) dont j'ai trouvé plusieurs exemplaires bien lisibles. J'interpréterais volontiers les deux points initiaux comme représentant les deux extrémités d'un $C$ sur un poinçon où la courbe de la lettre aurait été mal venue ou trop en retrait pour laisser son impression.

9. DIVIXI : Divixtus, Lezoux. période Trajan- $A$ ntonin.

10. GENIALI ... (ienialis, Lezoux, Hadrien-Antonin.

11. IVII.F. ( $F$ représenté par un trait et un demi-trait verticaux). Marque inconnue.

12. IVRI.I. Même marque à Poitiers sur forme Dragendorff 27. Date et atelier inconnus.

13. LCCI.F. Signalures identiques à Poitiers et à Périgueux. Lezoux, probablement époque flavienne.

14. MASCLOS.F. Masclus, La Graufesenque, Claude-Vespasien.

15. MAXMO et MAXMOS ( $M$ et $A$, liés, probablement aussi $I$ et $M$ ). Maximus, Lezoux, Hadrien-Antonin.

(6) Déchetette, Manuel, III, fig. 679 , p. $1485=990$. 
16. N.Lil CI.F. ( $F$ représenté par un trait et un demi-trail verticaux parallèles). I'ne marque identique lrouvée à Trion (Lyon). Sans lieu ni date.

17. OPIANIS.F. : inconnu d'Oswald.

18. ORANTIX. ( $N$ et $T$ liés). Exemplaires nombreux de cette signature. Le trait horizont al du $T$ touche le bord supérieur du cartouche; sur la plupart des exemplaires ce trait est peu visible. parfois indiscernable mais plusieurs exemplaires, mieux empreints ou mieux conservés, metlent la lecture hors de doute. Particulièrement fréquente sur des assiettes, coupes et bols 1)ragendorfr 29 ( I $^{\text {er }}$ siècle) $)$. Nom inconnu d'Oswald.

19. RIGIO M. Aucune marque à rapprocher.

20. SENISER... Seniserus a signé avec Ardacus des vases à Lezoux. Sans date.

21. XIIO : marque inconnue.

Les renvois d'Oswald a La Graufesenque n'ont rien de particulièrement étonnant: il a toujours été admis que des potiers de La Graufesenque avaient pu venir travailler a Lezoux. Je me suis trouvée plus étonnée des mentions de la fin du Ier et même du ri e siècle ; il m'avait semblé que les trouvailles dans le voisinage de mon four formaient un tout homogène; il est possible que des potiers de même nom se rencontrent à Lezoux à des époques différentes mais les tessons, dans le sol de Lezoux, sont si abondants qu'on ne peut répondre de rien. Il en est, dans mon jardin, qui peuvent provenir des fouilles anciennes de mon père. En raison du nombre et de la diversité des marques non déchiffrées trouvées dans mon four et son entourage immédiat, M. Fournier me conseille de renoncer au titre de Four d'Orantixus que j'indiquais au début de cet article. Ce serait, comme tous les autres exemples de Lezoux, un four collectif. Laissons lui donc, comme aux autres, son anonymat.

Mme A. Mathoxière-Plicqle (†), A. Cirenier. 